\title{
Calibração e aplicação do modelo MUSLE em uma microbacia hidrográfica nos Tabuleiros Costeiros brasileiros
}

\author{
Junior C. Avanzi ${ }^{1}$, Marx L. N. Silva ${ }^{1}$, Nilton Curi ${ }^{1}$, Carlos R. de Mello ${ }^{2}$ \& Sebastião Fonseca ${ }^{3}$
}

\begin{abstract}
RESUMO
A erosão hídrica é uma das principais preocupações relacionadas ao manejo de solos tropicais e o escoamento superficial o responsável pelo transporte e deposição desses sedimentos, razão por que se objetivou, com este estudo, ajustar um modelo semi-empírico às variáveis hidrológicas do escoamento superficial, aplicando-se a Equação Universal de Perdas de Solo Modificada - MUSLE, em uma microbacia hidrográfica ocupada por plantações de eucalipto e floresta nativa, na região dos Tabuleiros Costeiros, Aracruz, ES. As classes de solo ocorrentes na microbacia são o Argissolo Amarelo textura média/argilosa (PA1), Argissolo Amarelo moderadamente rochoso (PA8) e Plintossolo Háplico (FX). O trabalho foi conduzido no período de 1997 a 2004. Modelos para o deflúvio e vazão de pico foram ajustados, obtendo-se estimativas adequadas, podendo-se utilizá-los para simulação hidrológica. O modelo MUSLE mostrou-se apropriado para predições das perdas de solo na microbacia, recomendando-se os coeficientes "a" e "b" iguais a 14,96 e 0,59, respectivamente, com fator topográfico de 1,28.
\end{abstract}

Palavras-chave: modelagem, escoamento superficial, vazão de pico, perdas de solo

\section{Calibration and application of the MUSLE model in a small watershed of the Coastal Table Lands of Brazil}

\begin{abstract}
Water erosion is one of the main worries linked to tropical soil management, surface runoff being responsible for sediment transportation and bedding particles. The objective of this study was to adopt a semi-empirical hydrological model to surface runoff and peak discharge, applying the Modified Universal Soil Loss Equation (MUSLE) to a small watershed occupied by eucalyptus plantations and native forest, in the Coastal Table Land region, Aracruz, in the State of Espírito Santo, Brazil. Medium clayey texture Yellow Argisol (PA1), moderately rocky Yellow Argisol (PA8), and Haplic Plinthosol (FX) are the main soil classes. Hydrological data were monitored from 1997 to 2004. Models for surface runoff and peak discharge were adjusted, obtaining adequate estimates which can be used for hydrological simulation. The MUSLE model showed itself to be appropriate for soil losse prediction in the small watershed, the "a" and "b" coefficients recommended as being equal to 14.96 and 0.59 , respectively, with a topographical factor equal to 1.28 .
\end{abstract}

Key words: modeling, surface runoff, peak discharge, soil losses

DCS/UFLA, CP 3037, CEP 37200-000, Lavras, MG. Fone: (35) 3829-1251. E-mail: javanzi@gmail.com; marx@ufla.br; niltcuri@ufla.br

2 DEG/UFLA. Fone: (35) 3829-1386. E-mail: crmello@ufla.br

3 Aracruz Celulose S.A. Rodovia Aracruz, Barra do Riacho, Km 25, CP 331011, CEP 29197-000, Aracruz, ES. E-mail: sf@aracruz.com.br 


\section{INTRODUÇÃO}

O escoamento superficial, direto ou deflúvio, é a fase do ciclo hidrológico mais diretamente associada à erosão hídrica. Apesar do impacto das gotas de chuva desempenhar papel importante na desagregação das partículas do solo, é o escoamento superficial que promove o transporte das partículas em que, dependendo da carga de sedimento e do volume e velocidade do escoamento, poderá haver deposição no leito dos corpos d'água. Muitas práticas de controle da erosão são, na verdade, práticas de controle da velocidade e do volume de escoamento superficial (Pruski et al., 2001).

Para estudo do escoamento da água em bacias hidrográficas, geralmente se recorre às hidrógrafas, cujo comportamento do escoamento é visualizado (Pozzebon, 2000); conforme Santos et al. (2001), trata-se de registros gráficos de vazões de um corpo d’água em relação ao tempo, os quais refletem o comportamento hidrológico da bacia hidrográfica. A partir do estudo das hidrógrafas é possível separar o escoamento superficial direto do escoamento total determinando-se, assim, o deflúvio, a vazão de pico e os tempos de resposta da bacia hidrográfica às variações produzida pela chuva.

Os elementos que constituem uma bacia hidrográfica influenciam diretamente as respostas aos eventos de precipitação destacando-se, segundo Goldenfum (2001) e Righetto (1998), as suas unidades pedológicas, cobertura vegetal, características geomorfológicas (declividade, formato, área e rede de drenagem) e, também, características geológicas (Resende et al., 2007). Ambientalmente, pode-se dizer que a bacia hidrográfica é a unidade ecossistêmica e morfológica que melhor reflete os impactos das interferências antrópicas (Jenkins et al., 1994), tais como a ocupação das terras com atividades agrícolas e florestais (Ranzini \& Lima, 2002).

Neste contexto, a simulação de eventos hidrossedimentológicos permite otimização de tempo, aplicação e simulação imediata. Os modelos podem ser usados para estabelecer mais conhecimentos sobre os sistemas ambientais, tanto como meio de testar hipóteses como de ajuste de métodos para predizer o manejo desses ecossistemas (Beven, 1989; Grayson et al., 1992; Tucci, 1998).

A estimativa das perdas de solo por erosão é uma informação básica, para qualquer tomada de decisão em termos de uso e de manejo das terras, possibilitando a formulação de propostas de ações corretivas (Schertz, 1983). Neste sentido, Williams (1975), desenvolveu o modelo MUSLE (Equação Universal de Perdas de Solo Modificada) para prever a produção de sedimentos em pequenas e médias bacias hidrográficas, por meio das análises de chuvas individuais.

A MUSLE é uma modificação do modelo proposto por Wischmeier \& Smith (1965; 1978), em que o fator energia cinética da chuva (R) por um fator de escoamento (Williams, 1975), gerado com base no volume escoado e na vazão de pico. A MUSLE apresenta um grande potencial de aplicação no Brasil em virtude da simplicidade de sua estrutura, ao número relativamente baixo de parâmetros empregados e à facilidade de calibração e aplicação (Chaves, 1991); outro aspecto significativo está relacionado ao banco de dados re- lativamente grande, quando comparado a outros modelos, uma vez que a maioria dos fatores é obtida com base no modelo EUPS (Equação Universal de Perdas de Solo), amplamente difundido no Brasil.

Sendo assim se propôs determinar as variáveis hidrológicas do escoamento superficial ajustando-se modelos matemáticos para a estimativa do deflúvio e da vazão de pico dados estes aplicados na calibração e na estimativa de perdas de solo pelo modelo MUSLE, para uma microbacia hidrográfica ocupada por plantações de eucalipto e floresta nativa, na região dos Tabuleiros Costeiros, no município de Aracruz, ES.

\section{MATERIAL E MÉTODOS}

O estudo foi conduzido na área experimental da Aracruz Celulose S.A., localizada no município de Aracruz, ES, situada entre as coordenadas $19^{\circ} 35^{\prime}$ e $20^{\circ} 15^{\prime} \mathrm{S}$ e $40^{\circ} 00^{\prime} \mathrm{e}$ $40^{\circ} 20^{\prime}$ W de Greenwich; o clima da região é Aw, segundo a classificação de Köppen; a temperatura média anual é de $23{ }^{\circ} \mathrm{C}$, com precipitação média anual de $1.400 \mathrm{~mm}$ ocorrendo, nos meses de primavera e verão, 65 a 75\% do total anual precipitado e, em apenas 1 ou 2 meses, as precipitações mensais são menores que 60 mm (EMBRAPA, 2000).

A área da microbacia experimental é de 286 ha, dos quais 190 ha são de plantações de eucalipto e 86 ha estão cobertos por floresta nativa (Mata Atlântica) em estádio avançado de regeneração; os 10 ha restantes compreendem estradas florestais. As classes dominantes de solo são o Argissolo Amarelo textura média/argilosa (PA1) com 162 ha, Argissolo Amarelo moderadamente rochoso (PA8) com 100 ha e Plintossolo Háplico (FX), ocupando 24 ha. O PA1 e o FX apresentam relevo plano a suave ondulado, sendo que o PA1 ocorre no topo das elevações e o FX em locais ligeiramente côncavos distribuídos dentro das áreas de PA1; o PA8 apresenta relevo forte ondulado, ocorrendo na encosta.

As terras da microbacia apresentam declividade média de $13,68 \%$, com elevações variando de 53 m no terço a montante da drenagem, a menos de $11 \mathrm{~m}$, na proximidade da seção de controle. O comprimento do curso d'água principal é de $2.866 \mathrm{~m}$ e do total de drenos, $8.029 \mathrm{~m}$, sendo a declividade do canal principal de $0,94 \%$ e a densidade de drenagem igual a $2,81 \mathrm{~km} \mathrm{~km}^{-2}$ o perímetro da microbacia hidrográfica corresponde a $8.214 \mathrm{~m}$, com um coeficiente de compacidade (kc) igual a 1,37, considerado como de mediana propensão a vazões de pico elevadas.

Os dados foram coletados no período de 1997 a 2004 sendo que, para as medições da precipitação pluviométrica, foi utilizada estação climatológica automatizada, gerando dados a cada 30 min. A partir da precipitação monitorada foram gerados os hietogramas e, por meio de um vertedouro na saída da microbacia, aparelhado com linígrafo automático, monitoraram-se as alturas de lâmina d’água, a cada 30 min, produzindo-se os respectivos linigramas, que foram convertidos em hidrogramas com base na curva-chave da seção de controle da microbacia, através dos quais se realizou a separação do escoamento superficial direto do escoamento total determinando-se, assim, o deflúvio, a vazão de pico e os 
tempos de resposta que caracterizam hidrologicamente a microbacia. A separação do escoamento superficial direto foi realizada segundo metodologia proposta por Tucci (2004), em que, durante o evento, o comportamento do escoamento subterrâneo é considerado linear. Com a combinação hietograma-hidrograma, determinaram-se as precipitações efetivas que produziram os respectivos deflúvios e vazões de pico.

Os modelos para estimativa do deflúvio e da vazão de pico para a microbacia hidrográfica têm, como variáveis independentes, características da precipitação importantes para o comportamento do escoamento, destacando-se a precipitação total, em mm (P), a intensidade média da precipitação, em $\mathrm{mm} \mathrm{h}^{-1}(\mathrm{I})$, a intensidade máxima em $30 \mathrm{~min}$, em $\mathrm{mm} \mathrm{h}^{-1}$ (Im), duração da chuva, em h (Dc) e o tempo de pico da chuva, em h (ti). Essas variáveis, combinadas entre si, geram variáveis mais complexas e podem apresentar maior significância estatística. Para seleção das mais significativas pelo teste de "t" de Student, a nível de 5\%, aplicou-se o procedimento de regressão múltipla Backward; assim, foram ajustados modelos lineares por meio de regressão múltipla estimando-se os respectivos coeficientes das variáveis significativas, com base no método de mínimos quadrados; o programa utilizado foi o pacote estatístico SAS for Windows (SAS Institute, 1985).

Os parâmetros da Equação Universal de Perdas de Solo (EUPS), gerados por Martins (2005), nesta microbacia, foram ajustados para a Equação Universal de Perdas de Solo Modificada (MUSLE), sendo este modelo aplicável para avaliar perdas de solo provenientes de eventos de chuvas individuais (Williams, 1975). A MUSLE é expressa pela seguinte equação (Williams, 1975):

$$
\mathrm{A}=\mathrm{a} \times(\mathrm{D} \times \mathrm{Qp})^{\mathrm{b}} \times \mathrm{K} \times \mathrm{LS} \times \mathrm{CP}
$$

em que A: representa as perdas de solo (Mg), “a” e "b”: coeficientes de ajuste (calibração); K: erodibilidade média da bacia ( $\left.\mathrm{Mg} \mathrm{h} \mathrm{MJ}^{-1} \mathrm{~mm}^{-1}\right)$; LS: fator topográfico (adimensional); CP: fatores de cobertura do solo e práticas conservacionistas (adimensionais); D: deflúvio ou escoamento superficial direto $(\mathrm{mm})$ e Qp: vazão de pico $\left(\mathrm{m}^{3} \mathrm{~s}^{-1}\right)$. Efetuou-se a calibração dos coeficientes "a" e "b" com base em dados de monitoramento hidrológico do deflúvio e da vazão de pico, extraídos diretamente das hidrógrafas, correspondendo a 2/3 das informações de monitoramento; para isto, os coeficientes foram estimados com base em regressão linear, fazendo-se:

$$
\mathrm{Y}=\mathrm{aX}
$$

em que:

$$
\begin{gathered}
\mathrm{Y}=\frac{\mathrm{A}}{\mathrm{K} \times \mathrm{LS} \times \mathrm{CP}} \\
\mathrm{X}=(\mathrm{D} \times \mathrm{Qp})
\end{gathered}
$$

Os parâmetros “a” e "b” são obtidos por linearização da Eq. 2, conforme Johnson et al. (1985).

O fator erodibilidade do solo (K), foi obtido de Mar- tins (2005), que trabalhou e gerou este parâmetro para os solos da microbacia hidrográfica, sendo 0,007; 0,017 e 0,0004 Mg h MJ-1 $\mathrm{mm}^{-1}$ para o PA1, FX e PA8, respectivamente; o fator $\mathrm{K}$ para a microbacia foi obtido por meio da média ponderada da área de cada classe de solo, sendo este valor da ordem de 0,00545 $\mathrm{Mg} \mathrm{h} \mathrm{MJ}^{-1} \mathrm{~mm}^{-1}$.

O fator topográfico (LS), comprimento de rampa e grau de declive, foram obtidos através da equação proposta por Wischmeier \& Smith (1978):

$$
\mathrm{LS}=\left(\frac{\mathrm{Le}}{22,1}\right)^{\mathrm{m}} \cdot\left(0,065+0,0454 \cdot \mathrm{S}+0,0065 \cdot \mathrm{S}^{2}\right)
$$

sendo $\mathrm{m}=0,2$ para $\mathrm{S}<1 \% ; \mathrm{m}=0,3$ para $1 \% \leq \mathrm{S} \leq 3 \%$; $\mathrm{m}=0,4$ para $3 \%<\mathrm{S}<5 \%$; $\mathrm{m}=5$ para $\mathrm{S} \geq 5 \%$, $\mathrm{S}-$ declividade da encosta (\%) e Le - comprimento da encosta (m).

Os parâmetros S e Le utilizados na equação de Wischmeier \& Smith (1978), foram determinados pela equação de Williams \& Berndt (1977):

$$
\mathrm{S}=0,25 \cdot \mathrm{Z} \cdot\left(\mathrm{LC}_{25}+\mathrm{LC}_{50}+\mathrm{LC}_{75}\right) / \mathrm{A}
$$

em que Z: é o desnível entre a seção de controle e o ponto mais elevado da bacia (m); $\mathrm{LC}_{25}, \mathrm{LC}_{50}$ e $\mathrm{LC}_{75}$ : os comprimentos das curvas de nível a 25, 50 e 75\% de Z e A: a área da bacia $\left(\mathrm{m}^{2}\right)$.

$$
\mathrm{Le}=\frac{\mathrm{LC} \cdot \mathrm{LB}}{2 \cdot \mathrm{EP} \cdot\left(\mathrm{LC}^{2} \cdot \mathrm{LB}^{2}\right)^{0,5}}
$$

em que LC: é o somatório dos comprimentos das curvas de nível da bacia hidrográfica (m); LB: o somatório dos comprimentos das curvas de nível de base (m) e EP: o número de pontos extremos (aqueles que ocorrem quando um canal ou talvegue principal corta as curvas de nível da bacia).

Os valores de cobertura do solo e práticas conservacionistas (CP) também foram obtidos de Martins (2005); tais valores foram, para a cultura do eucalipto e mata nativa, de 0,0025 e 0,00013, respectivamente; para as áreas de estrada e carreadores, o fator CP foi considerado como igual a 1,0 e o fator CP, para a microbacia, foi obtido por meio da média ponderada da área de cada cobertura de solo, sendo este valor da ordem de 0,037 .

Utilizou-se para validação da modelagem hidrológica proposta, o coeficiente de Nash (Tucci, 1998):

$$
\mathrm{r}^{2}=1-\left(\left(\sum(\mathrm{y}-\overline{\mathrm{y}})^{2}-\Sigma(\mathrm{y}-\hat{\mathrm{y}})^{2}\right) / \sum(\mathrm{y}-\overline{\mathrm{y}})^{2}\right)
$$

em que y: é o deflúvio ou vazão de pico observado; ỹ é a média dos valores de deflúvio ou vazão de pico observados e ŷ: é o deflúvio ou vazão de pico estimado. Por este coeficiente, os modelos podem ser classificados como de ótima qualidade quando $\mathrm{r}^{2}>0,75$, boa qualidade quando $0,45<\mathrm{r}^{2}<0,75$ e ruim a regular quando $\mathrm{r}^{2}<0,45$.

Para avaliação da qualidade dos parâmetros da MUSLE, utilizou-se o quadrado médio do erro (QME), conforme Ferreira (2005): 


$$
\mathrm{QME}=\Sigma\left((\mathrm{y}-\hat{\mathrm{y}})^{2} / \hat{\mathrm{y}}\right) / \mathrm{n}
$$

em que y: é a perda de solo observada e ŷ: a perda de solo estimada.

O modelo também foi avaliado em termos da sua acurácia para eventos de precipitação não utilizados no seu processo de ajuste e calibração, ou seja, os outros $1 / 3$ dos dados, etapa esta de fundamental importância para validação e melhoria da qualidade dos modelos, qualificando-os para predição do comportamento hidrossedimentológico da microbacia, demonstrando sua sensibilidade e acurácia.

Obteve-se a quantificação da produção de sedimento na microbacia mediante coletas e análises de sedimento na água que passa pelo vertedouro, localizado na seção de controle da microbacia, segundo procedimentos recomendados por Santos et al. (2001).

\section{RESULTADOS E DISCUSSÃO}

Apresentam-se, na Tabela 1, os modelos desenvolvidos para predição das variáveis hidrológicas, deflúvio e vazão de pico, baseados nas características da precipitação, extraídas diretamente do hietograma, e seus parâmetros estatísticos, expressos pelo coeficiente de determinação, significância estatística dos parâmetros ajustados pelo teste t e pelo coeficiente de Nash.

Tabela 1. Modelos ajustados para as variáveis deflúvio e vazão de pico, para a microbacia hidrográfica estudada nos Tabuleiros Costeiros, ES

\begin{tabular}{|c|c|c|c|c|c|}
\hline \multicolumn{3}{|c|}{ Deflúvio } & \multicolumn{3}{|c|}{ Vazão de Pico } \\
\hline Variável & Parâmetro ${ }^{1}$ & Sig. Est. & Variável & Parâmetro ${ }^{1}$ & Sig. Est. \\
\hline Intercepto & 0,24213 & $0,0103^{*}$ & Intercepto & 4,78805 & $0,0341^{*}$ \\
\hline $\mathrm{P}^{2} \times \mathrm{Im}^{2}$ & $-2,22 \times 10^{-7}$ & $0,0028^{\star *}$ & PxI & $-0,163$ & $0,0050^{\star *}$ \\
\hline $\log (I)$ & 0,90087 & $0,0015^{\star \star}$ & Pxti & 0,1008 & $0,0001^{\star *}$ \\
\hline $\log (I \times P)$ & $-0,39443$ & $0,0002^{\star *}$ & $\mid m \times I$ & 0,22323 & $0,0001^{* *}$ \\
\hline $\mathrm{P}^{2}$ & $-0,00102$ & $0,0001^{* *}$ & Im xti & $-1,0477$ & $0,0001^{* *}$ \\
\hline $\mathrm{Im}^{2}$ & 0,00223 & $0,0001^{* *}$ & Ixti & 2,46942 & $0,0001^{* *}$ \\
\hline$P^{2}$ & 0,01201 & $0,0001^{* *}$ & $P^{2} \times 1$ & 0,003652 & $0,0001^{* *}$ \\
\hline ti2 & $-0,00215$ & $0,0312^{\star}$ & $\operatorname{Im}^{2} x \mid$ & $-0,003298$ & $0,0001^{* *}$ \\
\hline $\operatorname{Im} x \mid$ & $-0,01220$ & $0,0001^{* *}$ & $\mathrm{Im}^{2} \mathrm{xti}$ & 0,02405 & \\
\hline $\mathrm{P}^{2} \mathrm{x} \operatorname{Im}$ & $1,74 \times 10^{-5}$ & $0,0003^{\text {** }}$ & & & \\
\hline $\mathrm{P}^{2} \times \mathrm{I}$ & $8,606 \times 10^{-5}$ & $0,0001^{* *}$ & & & \\
\hline $\mathrm{P}^{2} \mathrm{xti}$ & 0,000104 & $0,0001^{* *}$ & & & \\
\hline $\mathrm{Im}^{2} \mathrm{xti}$ & $-0,00033$ & $0,0001^{* *}$ & & & \\
\hline $\mathrm{R}^{2}$ & 0,9206 & & & 0,9565 & \\
\hline Coef. Nash & 0,9237 & & & 0,9562 & \\
\hline
\end{tabular}

$\mathrm{R}^{2}$ coeficiente de determinação; Sig. Est. significância estatística; 1 parâmetros estimados; ** significativo a 5\%; * significativo a 1\%; P precipitação total $(\mathrm{mm})$; I intensidade média da precipitação $\left(\mathrm{mm} \mathrm{h}^{-1}\right)$; Im intensidade máxima em 30 minutos $\left(\mathrm{mm} \mathrm{h}^{-1}\right)$; ti tempo de pico da chuva (h)

Os modelos descritos na Tabela 1, apresentaram coeficientes elevados de determinação. O coeficiente de Nash caracterizou os modelos como de alta qualidade e havendo alta significância estatística das estimativas dos parâmetros a níveis de 1 e 5\%. É importante mencionar que, para modelagem do deflúvio, foi necessário um número maior de variáveis a fim de se obter coeficiente de determinação elevado e estatisticamente significativo; esta situação, por sua vez, não comprometeu os graus de liberdade da regressão tendo em vista o elevado número de eventos monitorados. Um detalhe adicional consiste no fato de que todos os parâmetros das variáveis foram altamente significativos com o procedimento Backward excluindo aqueles não significativos, bem como os que revelaram covariância entre as variáveis; esses atributos, conforme Ferreira (2005) e Haan (2002), atestam a acurácia dos modelos propostos.

Pode-se observar, na Figura 1, as dispersões dos valores estimados do deflúvio e da vazão de pico em torno da reta 1:1. Com a distância entre os pontos e a reta 1:1, tem-se a magnitude do erro dos valores estimados verificando-se concentrações de pontos em torno da reta $1: 1$, para ambas as variáveis, reforçando as características de boa precisão da modelagem proposta; adicionalmente, a modelagem mostrouse mais eficaz na predição dos maiores valores, tanto do deflúvio como da vazão de pico, verificada pela menor dispersão dos dados próximos aos valores mais elevados; portanto, precipitações que produzem os maiores deflúvios e
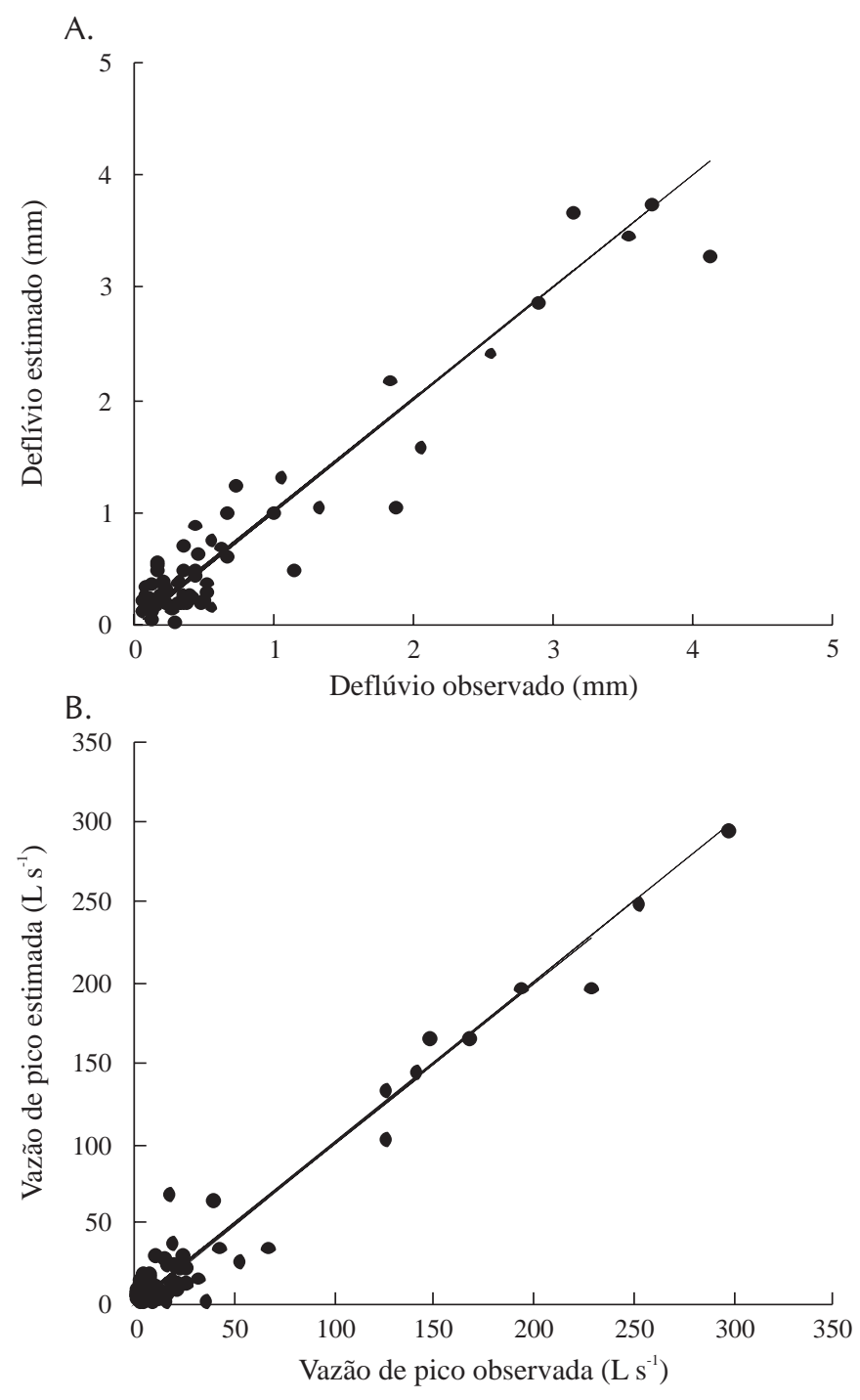

Figura 1. Dispersão dos valores estimados de deflúvio (A) e vazão de pico (B) pela modelagem semi-empírica, em torno da reta $1: 1$ 
vazões de pico e, conseqüentemente, as maiores perdas de sedimentos, foram modeladas com maior acurácia.

Pode-se visualizar, na Figura 2, os valores de precipitação que geraram os eventos de escoamento superficial no período de estudo, e se observar que as precipitações diárias se concentram no período de novembro a março, período em que se verifica maior ocorrência de chuvas e maior valor na umidade do solo. Esta associação das maiores quantidades de chuva, juntamente com os maiores valores de umidade do solo, proporciona os maiores deflúvios e vazões de pico, o que provocou os maiores arrastes de sedimentos na microbacia.

Através de regressão linear se ajustaram os coeficientes "a" e "b" da MUSLE, tendo-se obtido um valor igual a 14,96 e 0,59, respectivamente. Na Figura 3 se apresentam as dispersões dos valores das perdas de solo preditas pela
MUSLE, utilizando-se os dados não incluídos na geração dos parâmetros; percebe-se que o modelo calibrado apresentou grande erro de estimativa para um único evento de escoamento, sendo o quadrado médio do erro igual a 0,1646 Mg. Grande parte deste erro ocorreu em razão de dois eventos de precipitação muito semelhantes terem gerado, na microbacia, perdas de sedimento de dimensões bem distintas, podendo constituir-se, inclusive, em um erro de monitoramento do transporte de sedimentos o que, estatisticamente, é conhecido como outlier ou dado discrepante (Mello, 2003).

O evento de chuva verificado no dia 02/02/2002, produziu uma perda de solo no exutório de $0,729 \mathrm{Mg}$, ao passo que, em outro evento, com valores de vazão de pico e de deflúvio semelhantes, ocorrido no dia 18/07/2004, a perda de solo produzida foi de $0,141 \mathrm{Mg}$. A perda de solo constatada em fevereiro, foi

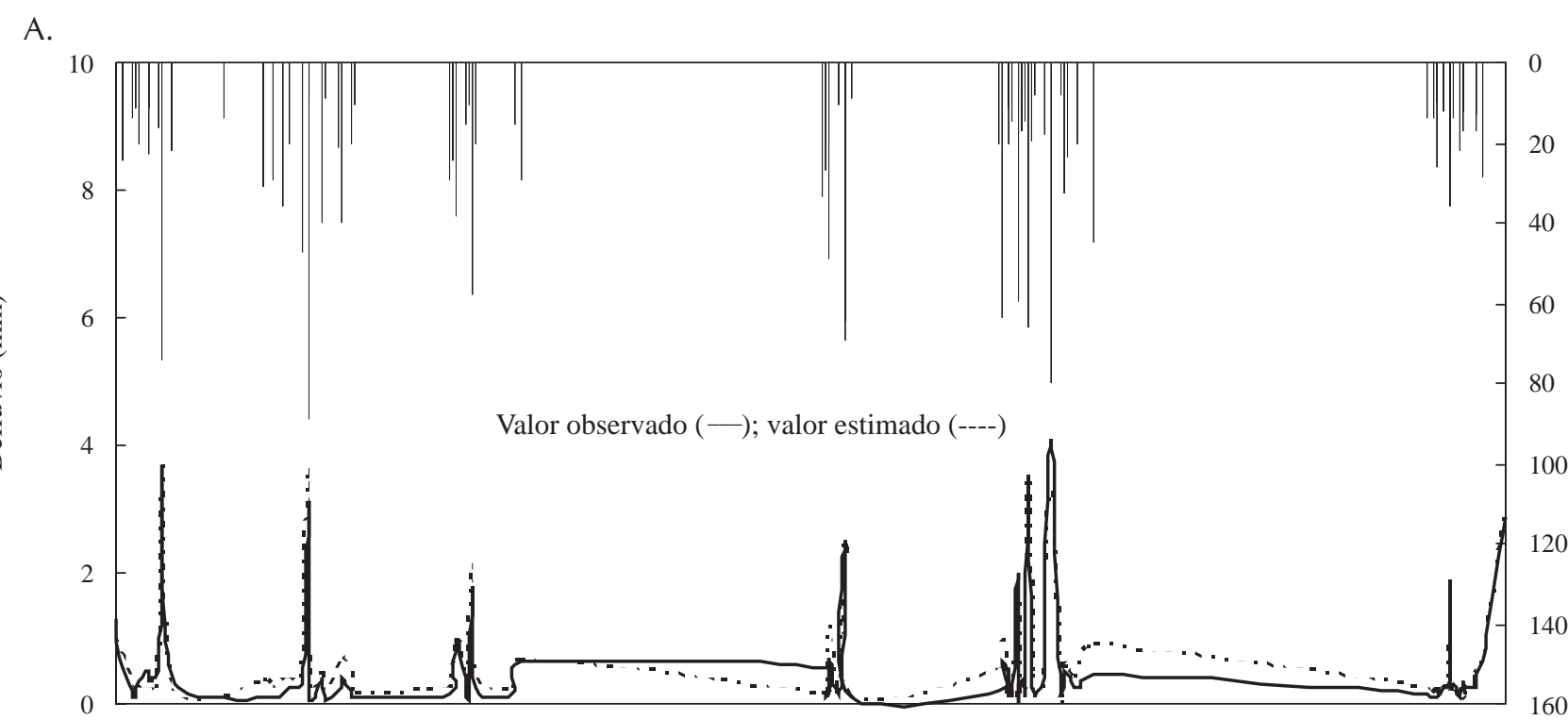

B.

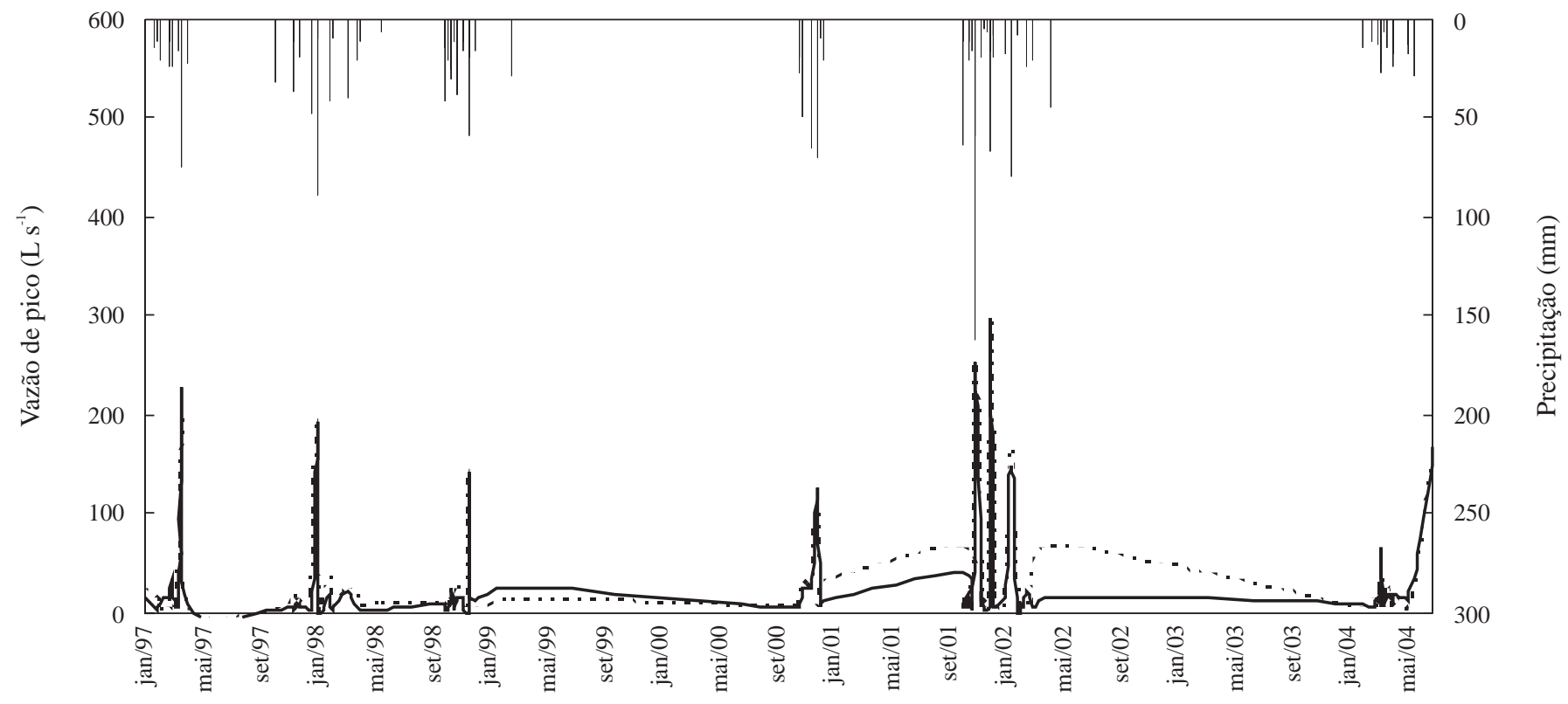

Figura 2. Precipitações e seus respectivos deflúvios (A) e vazões de pico (B) no período de 1997 a 2004, na microbacia estudada 

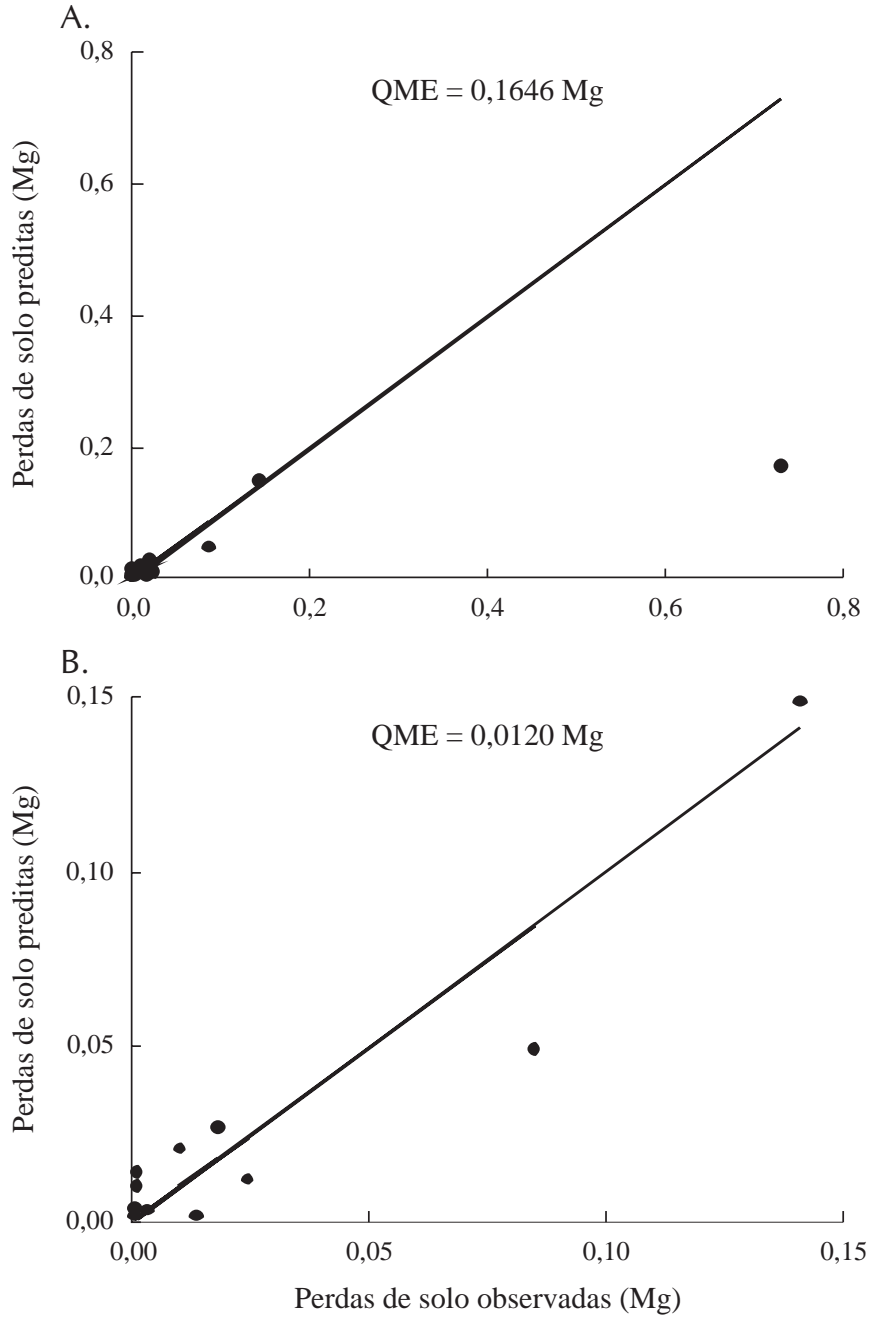

Figura 3. Valores de perda de solo estimados pela MUSLE utilizando-se os dados não incluídos na geração dos parâmetros " $a$ " $\mathrm{e}$ " $\mathrm{b}$ ". (A) todos os eventos estimados e (B) não se considerando o evento ocorrido em 02/02/2002

5,17 vezes maior que no evento de julho e o deflúvio gerado foi apenas 1,42 vezes maior; este erro pode ter ocorrido pelo fato dos modelos de predição de perdas de solo embasados na EUPS não levarem em consideração as condições de umidade antecedente do solo na determinação do fator erodibilidade; aspecto este que reforça que diferentes valores de umidade antecedente do solo podem induzir variações no fator erodibilidade. Estudos nos quais se analisa a influência da umidade na determinação do fator K para chuvas individuais foram realizados por Wischmeier (1976), constatando que eventos ocorridos em períodos extremos, solo muito seco ou muito saturado, provocam variações no valor de erodibilidade; caso o evento do dia 02/02/2002, que ocasionou grande valor de perdas de solo, não tivesse sido incluído na modelagem, a precisão do modelo MUSLE teria aumentado em mais de 10 vezes sendo que o QME nesta nova situação, seria de 0,0120 Mg (Figura 3B). O modelo MUSLE foi proposto para utilização de previsões de perdas de solo para chuvas individuais. Deste modo, um erro considerado em um único evento de chuva não compromete sua confiabilidade; além disso, o evento de chuva do dia 02/02/2002 pode ter sido influenciado por outros atributos não previstos pelo modelo MUSLE.
Pode-se observar, na Figura 4, os valores das perdas de solo estimados pela MUSLE, aplicando-se os modelos semiempíricos ajustados, para deflúvio e vazão de pico, descritos na Tabela 1, para os eventos de chuva não empregados na calibração da MUSLE.

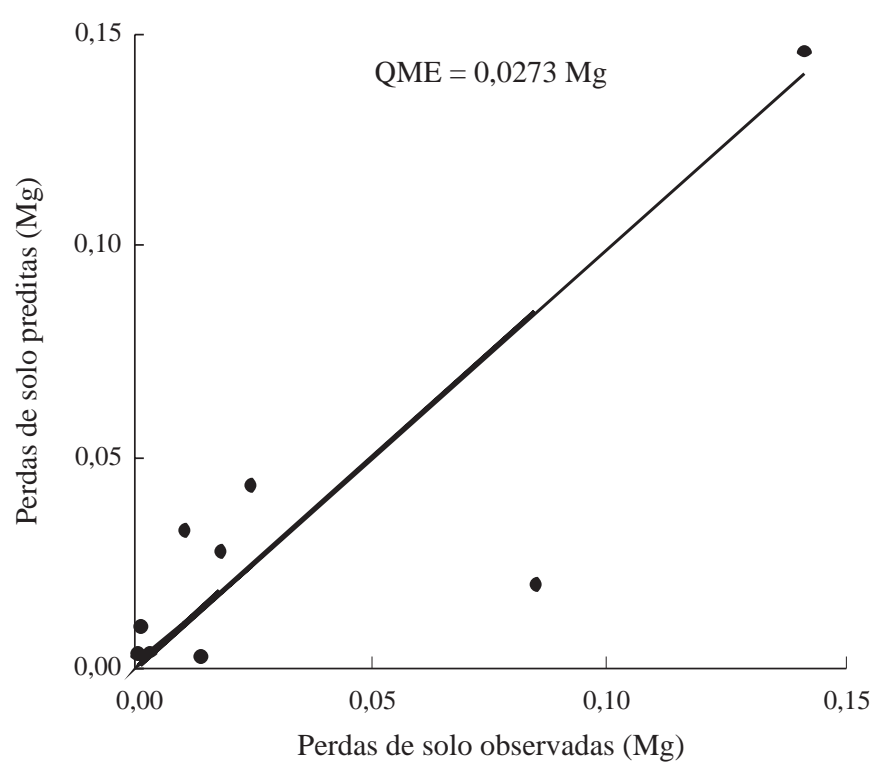

Figura 4. Perdas de solo observadas e preditas pela MUSLE utilizando-se a modelagem semi-empírica para a microbacia estudada nos Tabuleiros Costeiros, ES

Analisando-se a Figura 4, pode-se confirmar que a modelagem semi-empírica mostrou-se eficiente na predição dos parâmetros de escoamento, uma vez que os valores de perda de solo preditos pela MUSLE, para eventos não considerados em sua calibração, se mostraram consistentes e próximos dos valores observados, com valor do QME igual a 0,0273 Mg. Este baixo valor do QME mostra que, além da modelagem semi-empírica, a MUSLE também foi bem calibrada, pois este é resultado de todo o conjunto dos modelos desenvolvidos. Erskine et al. (2002), trabalhando com solos derivados de arenito na Austrália, avaliaram a produção de sedimento em pequenas bacias com o modelo MUSLE e encontraram coeficiente de correlação igual a 0,947 . Neste estudo ainda foram testados outros dois modelos (RUSLE e SoiLoss); contudo, o modelo MUSLE mostrou-se o de maior acurácia. Em estudos similares, realizados no oeste de Sidney, o modelo MUSLE registrou um coeficiente de correlação de 0,971 com os dados observados (Erskine et al., 2003).

\section{CONCLUSÕES}

1. A modelagem semi-empírica para o deflúvio e vazão de pico com base nas características da precipitação, mostrou boa acurácia, podendo ser aplicada para simulação dessas variáveis hidrológicas na microbacia estudada, visando ao seu planejamento ambiental.

2. O modelo MUSLE mostrou-se adequado para predições 
das perdas de solo na microbacia, recomendando-se os coeficientes "a" e "b” iguais a 14,96 e 0,59, respectivamente, com fator topográfico de 1,28.

3. A umidade antecedente do solo, embora não incluída nos modelos da EUPS/MUSLE, teve sua influência confirmada nos resultados desta pesquisa e deveria ser considerada em estudos futuros.

\section{LITERATURA CITADA}

Beven, K. Changing ideas in hydrology-the case of physically based models. Journal Hydrology, v.105, n.1/2, p.157-172, 1989.

Chaves, H. M. L. Análise global de sensibilidade dos parâmetros da Equação Universal de Perda de Solo Modificada (MUSLE). Revista Brasileira de Ciência do Solo, v.15, n.3, p.345-350, 1991.

EMBRAPA - Empresa Brasileira de Pesquisa Agropecuária. Centro Nacional de Pesquisa de Solos. Levantamentos generalizado e semidetalhado de solos da Aracruz Celulose S. A. no Estado do Espírito Santo e no estremo sul do Estado da Bahia e sua aplicação aos plantios de eucalipto. Rio de Janeiro: Embrapa, CNPS, 2000. parte 3, p.111.

Erskine, W. D.; Mahmoudzadeh, A.; Browning, C. M.; Myers, C. Sediment yields and soil loss rates from different land uses on Triassic shales in western Sydney, NSW. Australian Journal of Soil Research, v.41, n.1, p.127-140, 2003.

Erskine, W. D.; Mahmoudzadeh, A.; Myers, C. Land use effects on sediment yields and soil loss rates in small basins of Triassic sandstone near Sydney, NSW, Australia. Catena, v.49, n.4, p.271-287, 2002.

Ferreira, D. F. Estatística básica. Lavras: UFLA, 2005. 650p.

Goldenfum, J. A. Pequenas bacias hidrográficas: Conceitos básicos. In: Paiva, J. B. D.; Paiva, E. M. C. D. (ed.). Hidrologia aplicada à gestão de pequenas Bacias Hidrográficas. Porto Alegre: ABRH, 2001. p.3-14.

Grayson, R. B.; Moore, I. D.; McMahon, T. A. Physically-based hydrologic modeling: II. Is the concept realist? Water Resources Research, v.26, n.10, p.2659-2666, 1992.

Haan, C. T. Statistical methods in hydrology. 2.ed. Ames: Iowa State University Press, 2002. 377p.

Jenkins, A.; Peters, N. E.; Rodhe, A. Hydrology. In: Moldan, B.; Cerny, J. (ed.). Biogeochemistry of small catchments: A tool for environmental research. Chichester: John Wiley, 1994. p.31-54.

Johnson, C. W.; Gordon, N. D.; Hanson, C. L. Nothwest rangeland sediment yield analysis by the MUSLE. Transaction of the ASAE, v.8, n.6, p.1889-1895, 1985.

Martins, S. G. Erosão hídrica em povoamento de eucalipto sobre os solos coesos nos Tabuleiros Costeiros, ES. Lavras: UFLA, 2005, 106p. Tese Doutorado
Mello, C. R. Estudo hidrológico em microbacia hidrográfica com regime de escoamento efêmero. Lavras: UFLA, 2003, 133p. Tese Doutorado

Pozzebon, E. J. Simulação de escoamento superficial em microbacia hidrográfica utilizando sistema de informação geográfica e de modelagem hidrológica. Piracicaba: ESALQ, 2000, 243p. Tese Doutorado

Pruski, F. F.; Rodrigues, L. N.; Silva, D. D. Modelo hidrológico para estimativa do escoamento superficial em áreas agrícolas. Revista Brasileira de Engenharia Agrícola e Ambiental, v.5, n.2, p.301-307, 2001.

Ranzini, M.; Lima, W. P. Comportamento hidrológico, balanço de nutrientes e perdas de solo em duas microbacias reflorestadas com Eucalyptus, no Vale do Paraíba, SP. Scientia Florestalis, v.61, p.144-159, 2002.

Resende, M.; Curi, N.; Rezende, S. B.; Corrêa, G. F. Pedologia: Base para distinção de ambientes. 5.ed. Lavras: UFLA, 2007. 322p.

Righetto, A. M. Hidrologia e recursos hídricos. São Carlos: EESC/ USP, 1998. 840p.

Santos, I.; Fill, H. D.; Sugai, M. R. B.; Buda, H.; Kishi, R.; Marone, E.; Lautert, F. L. Hidrometria aplicada. Curitiba: LACTEC (Instituto de Tecnologia para o Desenvolvimento Centro Politécnico). 2001. 372p.

SAS Institute. SAS user's guide: Statistics. 5.ed. Cary, N. C. (ed), 1985. 956p.

Schertz, D. L. The basis for soil loss tolerance. Journal of Soil and Water Conservation, v.38, n.1, p.10-14, 1983.

Tucci, C. E. M. Modelos hidrológicos. Porto Alegre: ABRH/ UFRGS, 1998. 669p.

Tucci, C. E. M. Escoamento superficial. In: Tucci, C. E. M. (org.). Hidrologia: Ciência e aplicação. 3.ed. Porto Alegre: ABRH/ UFRGS, 2004. p.391-441.

Williams, J. R. Sediment-yield prediction with Universal Equation using runoff energy factor. In: Present and prospective technology for predicting sediment yield and sources. Oxford: USDA. ARS-S-40, 1975. p.244-252.

Williams, J. R.; Berndt, H. D. Determining the universal soil loss equation's length-slope factor for watershed. In: Soil erosion: Prediction and control. Ankeny: Soil Conservation Society of America, 1977. p.217-225.

Wischmeier, W. H. Use and misuse of the universal soil loss equation. Journal of Soil and Water Conservation, v.31, n.1, p.5-9, 1976.

Wischmeier, W. H.; Smith, D. D. Predicting rainfall erosion losses from cropland east of the Rocky Mountains: Guide for selection of practices for soil and water conservation. Washington: USDA, 1965. 47p. Agriculture Handbook, 282.

Wischmeier, W. H.; Smith, D. D. Predicting rainfall erosion losses: A guide to conservation planning. Washington: USDA, 1978. 58p. Agriculture Handbook, 537. 